\title{
Real-world outcomes of regorafenib combined with immune checkpoint inhibitors in patients with advanced or metastatic microsatellite stable colorectal cancer: A multicenter study
}

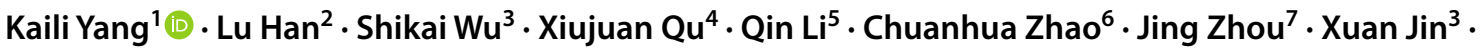 \\ Yusheng Wang ${ }^{8}$. Dong Yan ${ }^{9} \cdot$ Zhiqiang Cheng $^{10}$. Yuwei Hua ${ }^{1} \cdot$ Yan Zhang ${ }^{11} \cdot$ Yang Ge $^{12}$. Jinghua Sun ${ }^{13} \cdot$ Wei Deng $^{14}$. \\ Lin Zhao ${ }^{1}$. Yunbo Zhao ${ }^{15}$
}

Received: 24 June 2021 / Accepted: 4 October 2021 / Published online: 24 October 2021

(c) The Author(s) 2021

\begin{abstract}
Background Treatment strategies are limited for patients with chemotherapy refractory microsatellite stable (MSS) colorectal cancer. We aim to evaluate the efficacy and safety of immune checkpoint inhibitors (ICIs) combined with regorafenib in this population in routine clinical practice.

Methods We retrospectively analyzed patients with advanced or metastatic colorectal cancer who received at least one dose of ICIs combined with regorafenib in 14 Chinese medical centers. The primary outcome was objective response rate (ORR). This study was registered at ClinicalTrials.gov on February 2020 (NCT04771715).

Results Eighty-four patients received ICIs combined with regorafenib from January 2019 to January 2021. Most patients $(91 \%)$ received two or more systemic treatment lines before the study treatment. Seventy-six patients (90\%) had confirmed MSS status. At a median follow-up of 5.5 months, four patients achieved partial response (5\%) and 37 patients achieved stable disease (45\%) as the best response. The median progression-free survival (PFS) was 3.1 months, and the median overall survival was 17.3 months. Eleven patients (13\%) remained progression-free for more than 6 months. Baseline liver metastasis (HR 1.98, 95\%CI 1.07-3.69, $P=0.03$ ) and neutrophil-lymphocyte ratio (NLR) of $\geq 1.5$ (HR $2.83,95 \%$ CI 1.00-7.98, $P=0.05$ ) were associated with shorter PFS in multivariate analysis. Grade 3 or higher treatment-related adverse events (TRAEs) occurred in 16 patients (19\%).

Conclusion The combination of ICIs with regorafenib can be a valuable treatment option for a proportion of patients with chemotherapy refractory MSS colorectal cancer. Patients with no liver metastasis and a low NLR at baseline may derive most benefit from this strategy.
\end{abstract}

Keywords Colorectal cancer $\cdot$ Combination therapy $\cdot$ Immune checkpoint inhibitor $\cdot$ Regorafenib $\cdot$ Real world

\section{Introduction}

Colorectal cancer is the third most common cancer in the world with ascending incidence and mortality over the last decade [1]. Although the integration of targeted therapy into clinical practice has significantly increased the

Kaili Yang and Lu Han have contributed equally to this work.

Lin Zhao

wz20010727@aliyun.com

Yunbo Zhao

zhaoyb1206@163.com

Extended author information available on the last page of the article overall survival of patients with metastatic colorectal cancer (mCRC), treatment options after disease progression to standard of care are limited with modest survival benefit, and the long-term survival for chemotherapy refractory mCRC patients remains poor [2-4]. There is an unmet need for effective treatment strategies for these patients.

Immune checkpoint inhibitors (ICIs) have demonstrated promising efficacy in patients with microsatellite instabilityhigh (MSI-H) or mismatch repair-deficient (dMMR) mCRC [5-7]. However, MSI-H/dMMR tumors only account for $2-4 \%$ of the total mCRC cases [8]. Most colorectal cancer patients have a microsatellite stable (MSS) or mismatch repair proficient (pMMR) status and obtain little benefit from ICIs $[9,10]$. The difference of the tumor immune 
microenvironment between the two subtypes may account for the distinct response [11-13]. Thus, it is reasonable to apply combination strategies to modulate the microenvironment of MSS colorectal cancer and therefore exploit the benefit of ICIs. Regorafenib is a small molecule multi-kinase inhibitor that has been approved for treating chemotherapy refractory mCRC [14]. Besides its antiangiogenic effect, preclinical studies have demonstrated that regorafenib could modulate macrophage polarization and inhibit the expression of immunosuppressive molecules, which restored the immunosuppressive tumor microenvironment and synergistically enhanced the efficacy of ICIs [14-16]. A phase Ib trial has reported that the combination of nivolumab with regorafenib achieved an objective response rate of 33\% in patients with MSS/pMMR mCRC [17]. Meanwhile, in a recent phase II trial investigating avelumab combined with regorafenib, patients only achieved stable disease as the best response [18].

Therefore, the combination of ICIs with regorafenib may be a promising treatment strategy for patients with MSS/ pMMR mCRC. However, this strategy has been only applied in phase I or II trials with small sample sizes, where patients are usually hyperselected $[19,20]$. Whether this combination is effective for the heavily pretreated patients with multiple comorbidities in routine clinical practice remains unknown. To elucidate these issues, we conducted this retrospective study to evaluate the efficacy and safety of regorafenib combined with ICIs for patients with advanced or metastatic MSS colorectal cancer in the real world.

\section{Methods}

This retrospective study was conducted in 14 Chinese medical centers according to the Strengthening the Reporting of Observational Studies in Epidemiology (STROBE) reporting guideline [21]. The study was approved by the institutional review board of all participating centers and was registered at ClinicalTrials.gov (NCT04771715) on February 2021. Patients' consents for participation and publication were not required because of the retrospective design and the deidentified data of this study.

\section{Patients}

We reviewed electronic medical records to identify patients with advanced or metastatic colorectal cancer who received at least one dose of ICIs combined with regorafenib from January 2019 to January 2021. The types of ICIs, treatment doses and schedules were determined per investigator's decision. Previous exposure to ICIs or regorafenib was acceptable. Patients with confirmed MSI-H/dMMR status were excluded.

\section{Outcome}

The primary outcome of this study was objective response rate (ORR). Secondary outcomes included disease control rate (DCR), progression-free survival (PFS), overall survival (OS) and incidence of treatment-related adverse events (TRAEs). The responses were evaluated by local investigators per Response Evaluation Criteria in Solid Tumors version 1.1. The ORR was defined as the proportion of patients with complete response (CR) or partial response (PR) as their best response. The DCR was defined as the proportion of patients with CR, PR or stable disease (SD). The OS was defined as the time from treatment initiation to death from any cause. The PFS was defined as the time from treatment initiation to the first documented disease progression or death. TRAEs were evaluated according to the Common Terminology Criteria for Adverse Events version 4.0. Baseline characteristics of patients before the treatment initiation were collected for exploratory analyses. The MSI/MMR status was examined in each center by local investigators. The MMR status was determined by immunohistochemistry examining the expression of the four MMR enzymes (MLH1, MSH2, MSH6 and PMS2). The MSI status was determined by polymerase chain reaction assays examining the five microsatellite loci (BAT24, BAT26, D5S346, D2S123 and D17S250). The baseline neutrophil-lymphocyte ratio (NLR) was calculated from the baseline complete blood count (CBC) results if available.

\section{Statistical analysis}

We used R (version 4.0.3) to perform all statistical analyses. Patients were included for efficacy analysis if they had confirmed treatment discontinuation, available radiologic evaluation or at least eight weeks of follow-up after the initiation of study treatment. Patients with at least one available laboratory or vital sign measurement after the study treatment were included for safety analysis. We estimated the OS and PFS using the Kaplan-Meier method. We performed exploratory analyses on DCR and PFS to evaluate the potential effect of clinical variables on responses. The DCR was analyzed using logistic regression model, and the PFS was analyzed using Cox proportional hazard model. Univariate analysis and multivariate analysis adjusting for age, Eastern Cooperative Oncology Group Performance Status (ECOG PS), RAS mutation status and site of primary tumor were performed. The optimal cutoff value of NLR was defined using the maximally selected rank statistics method [22]. The proportional hazard (PH) assumption was tested by the Schoenfeld residuals test. All 
statistical tests were two-tailed, and $P<0.05$ was considered statistically significant. The incidence of TRAEs was analyzed in a descriptive method.

\section{Results}

\section{Patients}

A total of 84 patients were identified. Table 1 summarizes the baseline characteristics of patients at treatment initiation. The median age was 63 years (range, 35-81 years). The majority of the patients were male (60\%), had ECOG PS of 0 or $1(98 \%)$ and had tumors on the left side (76\%). Fifty-nine patients (70\%) had multiple metastatic sites; the most common metastatic sites included liver (65\%), lung (56\%) and lymph node (36\%). Forty-five patients (54\%) had KRAS or NRAS mutant tumors, and three patients $(4 \%)$ had BRAF ${ }^{\mathrm{V} 600 \mathrm{E}}$ mutations. The MSS/pMMR status was confirmed in 76 patients (90\%). Most patients (91\%) received two or more systemic treatment lines before the study treatment, and antiangiogenic treatment was previously used in 73 patients (87\%).

The characteristics of the study treatment are summarized in Supplementary Table 1 . The types of ICIs included sintilimab (39\%), nivolumab (20\%), toripalimab (15\%), camrelizumab (14\%), pembrolizumab (7\%) and tislelizumab (4\%). Among them, sintilimab, toripalimab, camrelizumab and tislelizumab were Chinese domestic ICIs. All ICIs were programmed cell death-1 (PD-1) inhibitors. Most patients (76\%) received regorafenib $80 \mathrm{mg}$ as the final dose. At a median follow-up of 5.5 months $(95 \%$ confidence interval [CI], 4.1-8.5), 15 patients (18\%) were still on treatment, while other patients discontinued the treatment because of disease progression (54\%), TRAEs (17\%) or other reasons (12\%). The median cycle of ICIs received was 4 (range, 1-24), and the median treatment duration was 4.3 months (range, 0.5-18.8).

\section{Efficacy}

A total of 82 patients were evaluable for response (Table 2). Two patients were excluded due to the lack of radiologic assessment. Four patients (5\%) achieved PR and 37 patients (45\%) achieved SD as the best response. Among the four patients achieving PR, the types of ICIs included nivolumab $(n=1)$, pembrolizumab $(n=1)$, sintilimab $(n=1)$ and camrelizumab $(n=1)$. The median duration of response was 5 months (range, 4.8-17.2), and two patients had ongoing responses at the time of analysis, including one patient with PR for 17.2 months. The median duration of disease control was 6.3 months (range, 0.5-17.2).
Table 1 Baseline characteristics of included patients $(n=84)$

\begin{tabular}{|c|c|}
\hline Characteristics & No. $(\%)$ \\
\hline No. of patients & 84 \\
\hline Median age, years (range) & $63(35-81)$ \\
\hline \multicolumn{2}{|l|}{ Age, years } \\
\hline$<70$ & $71(85)$ \\
\hline$\geq 70$ & $13(15)$ \\
\hline Male sex & $50(60)$ \\
\hline \multicolumn{2}{|l|}{ ECOG PS } \\
\hline 0 & $21(25)$ \\
\hline 1 & $61(73)$ \\
\hline 2 & $2(2)$ \\
\hline \multicolumn{2}{|l|}{ Site of primary tumor } \\
\hline Right-side colon & $20(24)$ \\
\hline Left-side colon and rectum & $64(76)$ \\
\hline Synchronous metastases & $49(58)$ \\
\hline \multicolumn{2}{|l|}{ Number of metastatic sites } \\
\hline Single & $25(30)$ \\
\hline Multiple & $59(70)$ \\
\hline \multicolumn{2}{|l|}{ Site of metastases } \\
\hline Lymph node & $30(36)$ \\
\hline Liver & $55(65)$ \\
\hline Lung & $47(56)$ \\
\hline Peritoneum & $18(21)$ \\
\hline Bone & $9(11)$ \\
\hline \multicolumn{2}{|l|}{ Mutation status } \\
\hline BRAF, KRAS, NRAS all wild type & $29(35)$ \\
\hline KRAS or NRAS mutant & $45(54)$ \\
\hline $\mathrm{BRAF}^{\mathrm{V} 600 \mathrm{E}}$ mutant $^{\mathrm{a}}$ & $3(4)$ \\
\hline Unkown & $8(10)$ \\
\hline \multicolumn{2}{|l|}{ MSS/pMMR status } \\
\hline Confirmed & $76(90)$ \\
\hline Unknown & $8(10)$ \\
\hline Median previous systemic treatment lines (range) & $3(0-8)$ \\
\hline \multicolumn{2}{|l|}{ Prior systemic treatment lines } \\
\hline 0 & $1(1)^{\mathrm{b}}$ \\
\hline 1 & $7(8)$ \\
\hline 2 & $25(30)$ \\
\hline 3 & $23(27)$ \\
\hline$\geq 4$ & $28(33)$ \\
\hline \multicolumn{2}{|l|}{ Prior systemic treatment regimens } \\
\hline Fluoropyrimidines & $82(98)$ \\
\hline Oxaliplatin & $72(86)$ \\
\hline Irinotecan & $72(86)$ \\
\hline Anti-EGFR treatment & $23(27)$ \\
\hline Anti-VEGF treatment & $73(87)$ \\
\hline Regorafenib & $22(26)$ \\
\hline PD-1 inhibitors & $3(4)$ \\
\hline \multicolumn{2}{|c|}{ Time from metastatic condition to study treatment initiation } \\
\hline$<18$ months & $43(51)$ \\
\hline$\geq 18$ months & $41(49)$ \\
\hline
\end{tabular}


Table 1 (continued)

\begin{tabular}{ll}
\hline Characteristics & No. (\%) \\
\hline Baseline NLR & \\
$<1.5$ & $10(12)$ \\
$\geq 1.5$ & $72(86)$ \\
Not applicable & $2(2)$ \\
\hline
\end{tabular}

${ }^{\mathrm{a}}$ One patient has both a BRAF ${ }^{\mathrm{V} 600 \mathrm{E}}$ mutation and a NRAS mutation

${ }^{\mathrm{b}}$ This patient received adjuvant chemotherapy after surgical resection of the primary tumor

ECOG PS Eastern Cooperative Oncology Group Performance Status; $E G F R$ epidermal growth factor receptor; $M S S$ microsatellite stable; $N L R$ neutrophil-lymphocyte ratio; $P D-1$ programmed cell death-1; pMMR mismatch repair proficient; VEGF vascular endothelial growth factor

Table 2 Antitumor activity in evaluable patients $(n=82)$

\begin{tabular}{ll}
\hline & No. $(\%)$ \\
\hline Best response & \\
Complete response & 0 \\
Partial response & $4(5)$ \\
Stable disease & $37(45)$ \\
Progressive disease & $41(50)$ \\
Overall response & $4(5)$ \\
Disease control & $41(50)$ \\
Median duration of response, months (range) & $5.0(4.8$ to $17.2+)$ \\
Median duration of disease control, months & $6.3(0.5$ to $17.2+)$ \\
(range) $^{\mathrm{b}}$ & \\
\hline
\end{tabular}

${ }^{\mathrm{a}}$ The Kaplan-Meier method for censored data was used to calculate the duration. The plus sign (+) indicates no progressive disease by the time of the last assessment

${ }^{b}$ Disease control was defined as complete response, partial response and stable disease

Univariate analysis of DCR revealed that liver metastasis (odds ratio [OR] 2.68, 95\% CI 1.06-7.06, $\mathrm{P}=0.04$ ), previous regorafenib treatment (OR 3.73, 95\% CI 1.33-11.65, $\mathrm{P}=0.02)$ and baseline NLR of $\geq 1.5$ (OR 5.03, 95\%CI 1.16-34.97, $\mathrm{P}=0.05)$ were associated with increased risk of disease progression (Fig. 1A). After adjusting for age, ECOG PS, RAS mutation status and primary tumor sidedness, the effect of liver metastasis (OR 3.80, 95\% CI 1.33-11.76, $\mathrm{P}=0.02$ ) and previous regorafenib treatment (OR 3.62, 95\%CI 1.12-13.28, $\mathrm{P}=0.04$ ) remained statistically significant (Supplementary Table 2). Factors including other previous treatment regimens did not affect the DCR.

All 84 patients were evaluable for OS, and one patient was excluded from PFS analysis due to the lack of radiologic assessment. The median PFS was 3.1 months $(95 \% \mathrm{CI}$, 2.3-4.2) (Fig. 2A), and the median OS was 17.3 months (95\% CI, 11.3-not reached) (Fig. 2B). A total of 29 patients (35\%) obtained PFS of $\geq 3$ months, and 11 patients (13\%) obtained PFS of $\geq 6$ months. In the univariate analysis, $\geq 4$ metastatic sites (hazard ratio [HR] 2.76, 95\%CI 1.38-5.52, $\mathrm{P}=0.004)$ and baseline NLR of $\geq 1.5$ (HR $3.43 ; 95 \% \mathrm{CI}$ 1.24-9.54, $\mathrm{P}=0.02$ ) were associated with shorter PFS (Fig. 1B). Multivariate analysis revealed that $\geq 4$ metastatic sites (HR 1.35, 95\% CI 1.05-1.73, $\mathrm{P}=0.02$ ), liver metastasis (HR 1.98, 95\% CI 1.07-3.69, $\mathrm{P}=0.03$ ) and baseline NLR of $\geq 1.5$ (HR $2.83,95 \%$ CI $1.00-7.98, \mathrm{P}=0.05$ ) were associated with shorter PFS. Other factors, including previous ICIs treatment and previous antiangiogenic treatment, did not affect the PFS.

The $\mathrm{PH}$ assumption was satisfied for all clinical variables included in the analysis (Supplementary Table 3).

\section{Safety}

All 84 patients were evaluable for safety. Fifty-one patients (61\%) experienced TRAEs during the treatment, and 16 patients (19\%) experienced grade 3 TRAEs (Table 3). The most common TRAEs included fatigue $(21 \%)$, rash $(15 \%)$ and hand-foot skin reaction (14\%); the most common grade 3 TRAEs included rash (7\%), hand-foot skin reaction (4\%), thrombocytopenia (2\%) and myocardial enzyme elevation (2\%). One patient experienced treatment-related death because of grade 5 myasthenia gravis. A total of 22 patients (26\%) had regorafenib dose reduction or treatment termination because of TRAEs. Among 67 patients who received regorafenib $80 \mathrm{mg}$ or less as the initial dose, 38 patients (57\%) experienced TRAEs, including 10 patients (15\%) with grade 3 TRAEs; two patients had dose reduction and nine patients discontinued the treatment because of TRAEs. For 17 patients who received regorafenib $120 \mathrm{mg}$ or more as the initial dose, the number of all grade and grade 3 TRAEs were $12(71 \%)$ and five $(29 \%)$; six patients had dose reduction and five patients discontinued the treatment because of TRAEs. Among 13 patients older than 70 years, eight patients experienced TRAEs (62\%), and three patients experienced grade 3 TRAEs (23\%).

\section{Discussion}

The long-term prognosis of mCRC remains dismal in part because of the lack of effective treatment strategies beyond progression to standard of care [2]. Although recent advances of ICIs have demonstrated remarkable efficacy in patients with MSI-H status, the majority of colorectal cancer patients do not benefit from ICIs due to the immunosuppressive tumor microenvironment of MSS tumors [12, 23]. Currently, many studies are investigating combination strategies to reverse the immunosuppressive microenvironment of MSS colorectal cancer and therefore to exploit the long-term survival benefit of ICIs [12]. 


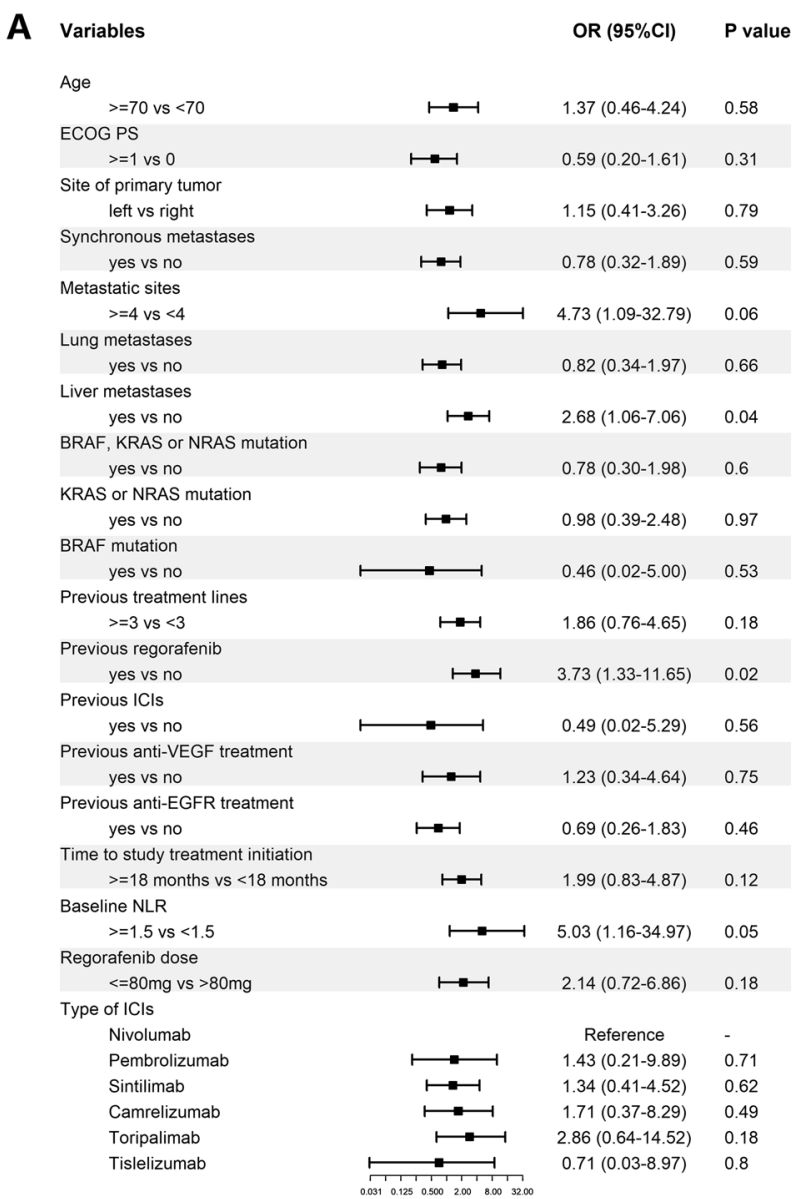

Fig. 1 Forest plot of univariate analysis for (A) disease control rate and (B) progression-free survival CI Confidence interval; ECOG PS Eastern Cooperative Oncology Group Performance Status; EGFR

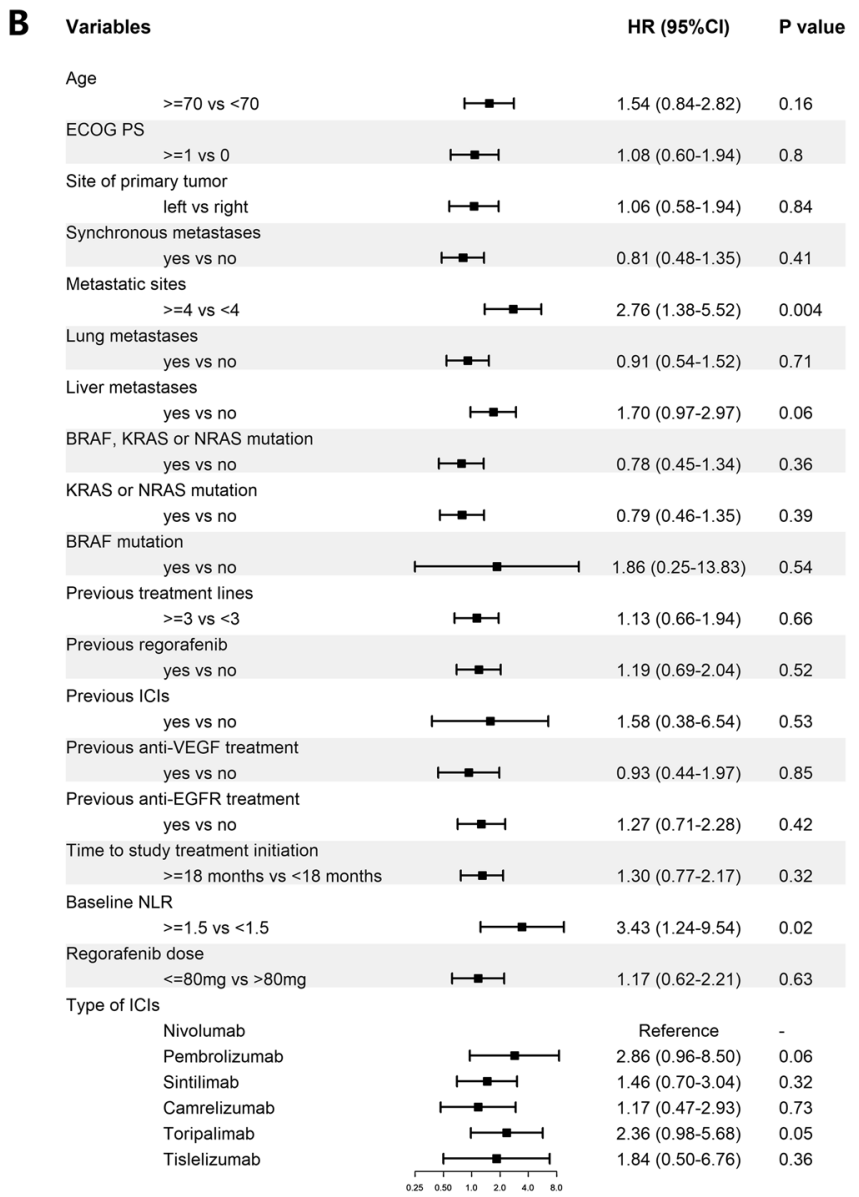

epidermal growth factor receptor; $H R$ hazard ratio; ICI immune checkpoint inhibitor; NLR neutrophil-lymphocyte ratio; $O R$ odds ratio; VEGF vascular endothelial growth factor

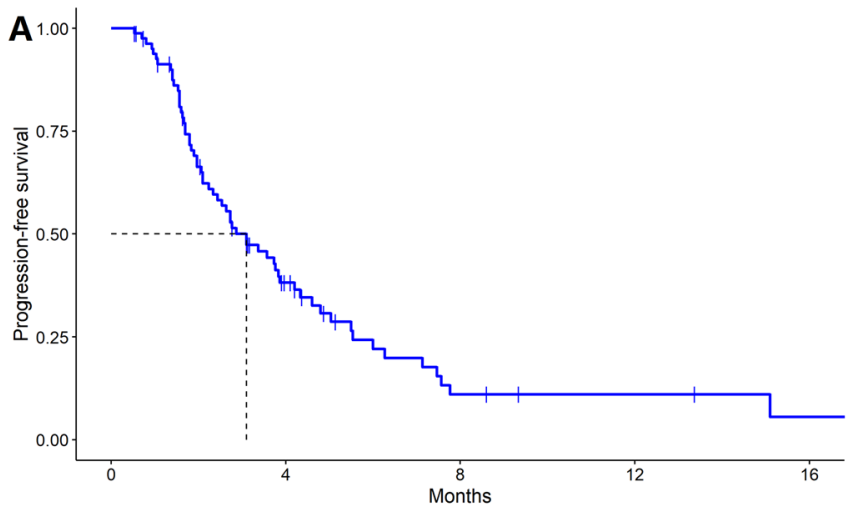

Number at risk

23

3

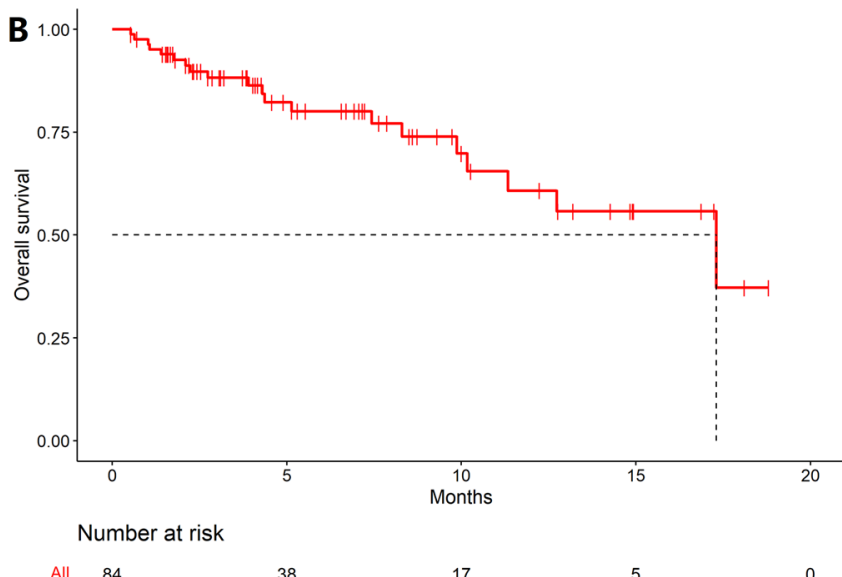

All $\quad 84 \quad 38 \quad 17$

Fig. 2 Kaplan-Meier curves of (A) progression-free survival $(n=83)$ and $(\mathbf{B})$ overall survival $(n=84)$ in patients with colorectal cancer treated with immune checkpoint inhibitors plus regorafenib 
Table 3 Incidence of treatment-related adverse events $(n=84)$

\begin{tabular}{lll}
\hline TRAEs & Any grade, no. (\%) & Grade $\geq 3$, no. (\%) \\
\hline All & $51(61)$ & $16(19)$ \\
Fatigue & $18(21)$ & 0 \\
Rash & $13(15)$ & $6(7)$ \\
Hand-foot skin reaction & $12(14)$ & $3(4)$ \\
Hypertension & $8(10)$ & $1(1)$ \\
Fever & $8(10)$ & 0 \\
Hypothyroidism & $7(8)$ & 0 \\
Transaminase elevation & $7(8)$ & 0 \\
Diarrhea & $6(7)$ & 0 \\
Anorexia & $4(5)$ & $1(1)$ \\
Oral mucositis & $4(5)$ & 0 \\
Myocardial enzyme eleva- & $3(4)$ & $2(2)$ \\
tion & & \\
Thrombocytopenia & $3(4)$ & $2(2)$ \\
Hoarseness & $3(4)$ & 0 \\
Myositis & $3(4)$ & 0 \\
Pancreatitis & $2(2)$ & 0 \\
Vomiting & $2(2)$ & 0 \\
Hematuria & $1(1)$ & $1(1)$ \\
Myasthenia gravis & $1(1)$ & $1(1)$ \\
Neutropenia & $1(1)$ & $1(1)$ \\
Visual field loss & $1(1)$ & $1(1)$ \\
Anemia & $1(1)$ & 0 \\
Arthralgia & $1(1)$ & 0 \\
Hyperthyroidism & $1(1)$ & $1(1)$ \\
Proteinuria & & 0 \\
\hline
\end{tabular}

TRAEs Treatment-related adverse events

In this study, we retrospectively analyzed the efficacy and safety of ICIs combined with regorafenib in patients with MSS colorectal cancer. To our knowledge, this is the largest cohort to evaluate the efficacy and safety of such combination strategy in real-world clinical practice. Our study demonstrated a modest ORR and PFS. Half of the patients could achieve disease control and a proportion of patients could remain progression-free for more than 6 months. This observation corresponded to the long-term survival benefit pattern of ICIs. Conventional treatment options for chemotherapy refractory colorectal cancer included regorafenib single agent and TAS-102 (trifluridine/tipiracil) [2, 24]. For patients whose disease progressed after antiangiogenic treatment, the median PFS was around 2 months and the median OS was 7 months [3, 4, 25]. The results of our study compared favorably with the conventional treatment. Thus, the combination of ICIs with regorafenib could be a feasible treatment option for chemotherapy refractory MSS colorectal cancer.

Several previous studies with small sample sizes have evaluated the efficacy of ICIs combined with regorafenib in
MSS colorectal cancer [17, 18, 26]. The results of our study were comparable with the REGOMUNE (NCT03475953) and the REGOTORI study (NCT03946917) $[18,26]$. However, in the phase Ib REGONIVO trial (NCT03406871), an ORR of $33 \%$ and a DCR of $88 \%$ were reported in 24 patients with MSS colorectal cancer, which was higher than the responses reported in other studies [17]. Several factors could account for the difference. First, the types of ICIs were different. The REGONIVO trial only investigated the combination of nivolumab with regorafenib, while patients could receive a variety of ICIs in real-world clinical practice. ICIs other than nivolumab were also analyzed in our study, including several Chinese domestic drugs such as sintilimab and toripalimab. All ICIs were PD-1 inhibitors in this study. Previous studies have suggested superior efficacy of PD-1 inhibitors than programmed cell death ligand-1 (PDL1) inhibitors, while the difference among different PD-1 inhibitors has not been fully elucidated [27]. Currently, only nivolumab and pembrolizumab have been approved for treating MSI-H colorectal cancer [28]. Although exploratory analyses detected no significant difference in response and PFS among different PD-1 inhibitors in this study, the heterogenous regimens may exhibit different treatment efficacy and require more investigations. Second, the baseline characteristics of patients were different. In the REGONIVO study, all patients had an ECOG PS of 0, and most patients did not bear RAS mutations. These participants represented a subset of patients with good prognosis and they could not reflect the population in routine clinical practice. Third, although the incidence of TRAEs was similar between two studies, only two patients (4\%) discontinued the treatment because of TRAEs in the REGONIVO trial, while this number was 14 $(17 \%)$ in our study. This difference may attribute to the fact that patients in the real world had generally poorer performance status and more comorbidities and requires cautious TRAEs management $[29,30]$. However, patients may not receive adequate treatment due to early treatment discontinuation, and thus, the efficacy could be underestimated in the real world. In summary, current evidence has demonstrated promising efficacy of ICIs combined with regorafenib in a subset of patients with MSS colorectal cancer, and further investigations should focus on patient selection.

To help patient selection, we performed exploratory analyses to identify clinical characteristics related to the efficacy of ICIs. Multivariate analysis revealed that liver metastasis was associated with inferior response and PFS. The presence of liver metastasis has been identified as an independent poor prognostic factor for multiple cancer types and particularly for ICIs treatment [31, 32]. More specifically, this association was preserved in clinical trials investigating the combination of regorafenib with ICIs in MSS colorectal cancer. In the REGONIVO trial, compared with the ORR of the entire MSS cohort (33\%), only two of 13 patients with liver 
metastasis (15\%) responded to the study treatment [17]. Our result was consistent with previous findings, further confirming the negative predictive role of liver metastasis. The microenvironment of liver metastasis was generally regarded immunosuppressive characterized by decreased infiltration of CD8 + T cells and functional enrichment of immune escape pathways [31, 33, 34]. Moreover, recent studies have revealed that liver metastases could induce systemic resistance to ICIs mediated by macrophages and regulatory $\mathrm{T}$ cells $[35,36]$. Therefore, effective management of liver metastases could be the key point to overcome the resistance to ICIs.

Our analysis also revealed that a high baseline NLR was associated with inferior PFS. The negative predictive role of NLR has been validated in many studies across different cancer types, and a recent study again confirmed its value in a large cohort of 1714 patients receiving ICIs [37-39]. However, NLR could be affected by factors other than cancer progression, such as infection, steroids use and preexisting autoimmune disease, which limits its value as a cancer predictive biomarker. Besides, current application of NLR lacks a standardized threshold. The cutoff value between the high and the low NLR varied from 1.9 to 7.2 in previous publications and was calculated using certain statistical methods without biological significance [40]. Currently, the dynamic change of peripheral immune cell content during cancer progression and ICIs treatment has not been fully elucidated. Previous studies have suggested that certain neutrophil subsets in the peripheral blood conferred the immunosuppressive ability [41, 42]. Further inspection into the neutrophil heterogeneity may improve the predictive value of NLR. Moreover, for biomarkers other than NLR, a previous study demonstrated superior predictive value of the combination of NLR and TMB than NLR alone [39]. In a small cohort investigating ICIs combined with regorafenib in MSS colorectal cancer, the dynamic change of circulating tumor DNA during early treatment correlated with the response [18]. Predictive models integrating multiple parameters may serve as valuable biomarkers and augment patient selection for the combination strategy.

The toxicity profile of this study was generally tolerable and was comparable with previous studies evaluating the same strategy; the incidence of TRAEs was also similar to conventional treatment such as regorafenib single agent or TAS-102 [3, 4, 17, 18, 26]. Thus, the combination of ICIs with regorafenib could be safely applied in patients with chemotherapy refractory MSS colorectal cancer. Noteworthily, some patients discontinued treatment because of TRAEs. This proportion was similar to the REGOMUNE trial [18]. As what has been mentioned above, early treatment discontinuation may lead to inadequate treatment, and cautiousness should be paid to balance the benefit and risk. Currently, the optimal decision after treatment interruption because of TRAEs remained unclear [43]. In order to fully exploit the benefit of ICIs, retreatment after resolution of TRAEs is a feasible option $[44,45]$.

This study has several limitations. The major limitation is its retrospective design, which limits the applicability of the results. Second, the median follow-up is relatively short and the OS result remains immature, as half of the events have not occurred at the data cutoff, which may introduce potential confounders to the result. Thus, we only performed exploratory analyses on PFS and DCR to avoid misinterpretations. Third, the MSS status is not available in a small proportion of patients. However, considering that MSI-H/ dMMR tumors only account for $2-4 \%$ of total mCRC cases, this limitation may not introduce much bias [8].

\section{Conclusion}

In conclusion, the combination of ICIs with regorafenib provides a feasible treatment option for a proportion of patients with chemotherapy refractory MSS colorectal cancer. Prospective validations of this strategy in large cohorts are required, and further inspections into biological rationales may help identify the population who can derive most benefit from this strategy.

Supplementary Information The online version contains supplementary material available at https://doi.org/10.1007/s00262-021-03083-3.

Authors' contributions LZ, KLY, YBZ and LH were involved in conceptualization. LH, YBZ, XJQ, SKW, QL, CHZ, LZ, KLY, YSW, JZ, DY, ZQC, YZ, YG, JHS, WD and YWH had contributed to data acquisition. KLY, LH, LZ and YBZ carried out data analysis and interpretation and critically revised the manuscript. KLY and LH wrote the original draft.

Funding This work was supported by grants from the National Natural Science Foundation of China (No. 61435001) and the CAMS Innovation Fund for Medical Sciences (No. 2016-I2M-1-001, No. 2017-I2M-4-003).

Data availability All data are available upon reasonable request.

\section{Declarations}

Conflict of interest The authors declared no competing interests.

Ethics approval The study was approved by the institutional review board and ethics committee of all participating centers, and all the procedures being performed were part of the routine care. This study was registered on ClinicalTrials.gov on February 2020 (NCT04771715).

Open Access This article is licensed under a Creative Commons Attribution 4.0 International License, which permits use, sharing, adaptation, distribution and reproduction in any medium or format, as long as you give appropriate credit to the original author(s) and the source, provide a link to the Creative Commons licence, and indicate if changes were made. The images or other third party material in this article are 
included in the article's Creative Commons licence, unless indicated otherwise in a credit line to the material. If material is not included in the article's Creative Commons licence and your intended use is not permitted by statutory regulation or exceeds the permitted use, you will need to obtain permission directly from the copyright holder. To view a copy of this licence, visit http://creativecommons.org/licenses/by/4.0/.

\section{References}

1. Sung $\mathrm{H}$ et al (2021) Global cancer statistics 2020: GLOBOCAN estimates of incidence and mortality worldwide for 36 cancers in 185 countries. CA Cancer J Clin 71(3):209-249

2. Modest DP, Pant S, Sartore-Bianchi A (2019) Treatment sequencing in metastatic colorectal cancer. Eur J Cancer 109:70-83

3. Grothey A et al (2013) Regorafenib monotherapy for previously treated metastatic colorectal cancer (CORRECT): an international, multicentre, randomised, placebo-controlled, phase 3 trial. The Lancet 381(9863):303-312

4. Mayer RJ et al (2015) Randomized trial of TAS-102 for refractory metastatic colorectal cancer. N Engl J Med 372(20):1909-1919

5. Overman MJ et al (2017) Nivolumab in patients with metastatic DNA mismatch repair-deficient or microsatellite instability-high colorectal cancer (CheckMate 142): an open-label, multicentre, phase 2 study. Lancet Oncol 18(9):1182-1191

6. Le DT et al (2017) Mismatch repair deficiency predicts response of solid tumors to PD-1 blockade. Science 357(6349):409-413

7. André $\mathrm{T}$ et al (2020) Pembrolizumab in microsatelliteinstability-high advanced colorectal cancer. N Engl J Med 383(23):2207-2218

8. Boland CR et al (1998) A National Cancer Institute Workshop on Microsatellite Instability for cancer detection and familial predisposition: development of international criteria for the determination of microsatellite instability in colorectal cancer. Cancer Res 58(22):5248-5257

9. Lee JJ et al (2017) Phase 2 study of pembrolizumab in combination with azacitidine in subjects with metastatic colorectal cancer. J Clin Oncol 35(15_Suppl):3054-3054

10. Eng C et al (2019) Atezolizumab with or without cobimetinib versus regorafenib in previously treated metastatic colorectal cancer (IMblaze370): a multicentre, open-label, phase 3, randomised, controlled trial. Lancet Oncol 20(6):849-861

11. Guinney J et al (2015) The consensus molecular subtypes of colorectal cancer. Nat Med 21(11):1350-1356

12. Ciardiello D et al (2019) Immunotherapy of colorectal cancer: Challenges for therapeutic efficacy. Cancer Treat Rev 76:22-32

13. Pagès $F$ et al (2018) International validation of the consensus Immunoscore for the classification of colon cancer: a prognostic and accuracy study. Lancet 391(10135):2128-2139

14. Arai H et al (2019) 2019 Molecular insight of regorafenib treatment for colorectal cancer. Cancer Treat Rev 81:101912

15. Hoff S et al (2017) 1198P - Immunomodulation by regorafenib alone and in combination with anti PD1 antibody on murine models of colorectal cancer. Ann Oncol 28:423

16. Wu RY et al (2019) Regorafenib promotes antitumor immunity via inhibiting PD-L1 and IDO1 expression in melanoma. Clin Cancer Res 25(14):4530-4541

17. Fukuoka S et al (2020) Regorafenib plus nivolumab in patients with advanced gastric or colorectal cancer: an open-label, doseescalation, and dose-expansion phase Ib Trial (REGONIVO, EPOC1603). J Clin Oncol 38(18):2053-2061

18. Cousin S et al (2021) Regorafenib-Avelumab Combination in Patients with Microsatellite Stable Colorectal Cancer
(REGOMUNE): A Single-arm, Open-label. Phase II Trial. Clin Cancer Res 27(8):2139-2147

19. Shi, J.F., et al., Clinical characteristics, medical service utilization, and expenditure for colorectal cancer in China, 2005 to 2014: Overall design and results from a multicenter retrospective epidemiologic survey. Cancer, 2021.

20. Unger JM et al (2019) Association of Patient Comorbid Conditions With Cancer Clinical Trial Participation. JAMA Oncol 5(3):326-333

21. Vandenbroucke, J.P., et al., Strengthening the Reporting of Observational Studies in Epidemiology (STROBE): explanation and elaboration. PLoS Med, 2007. 4(10): p. e297.

22. Lausen B, Schumacher M (1992) Maximally Selected Rank Statistics. Biometrics 48(1):73-85

23. Morse MA, Hochster H, Benson A (2020) Perspectives on Treatment of Metastatic Colorectal Cancer with Immune Checkpoint Inhibitor Therapy. Oncologist 25(1):33-45

24. Yoshino T et al (2018) Pan-Asian adapted ESMO consensus guidelines for the management of patients with metastatic colorectal cancer: a JSMO-ESMO initiative endorsed by CSCO, KACO, MOS. SSO and TOS Ann Oncol 29(1):44-70

25. Li J et al (2015) Regorafenib plus best supportive care versus placebo plus best supportive care in Asian patients with previously treated metastatic colorectal cancer (CONCUR): a randomised, double-blind, placebo-controlled, phase 3 trial. Lancet Oncol 16(6):619-629

26. Wang F et al (2020) 433P A phase Ib/II clinical trial of tolerability, safety and efficacy of regorafenib in combination with toripalimab (a PD-1 antibody) in patients with relapsed or metastatic colorectal cancer. Ann Oncol 31:S425

27. Duan J et al (2020) Use of Immunotherapy With Programmed Cell Death 1 vs Programmed Cell Death Ligand 1 Inhibitors in Patients With Cancer: A Systematic Review and Meta-analysis. JAMA Oncol 6(3):375-384

28. Zhao Y, Liu L, Weng L (2021) Comparisons of Underlying Mechanisms, Clinical Efficacy and Safety Between Anti-PD-1 and AntiPD-L1 Immunotherapy: The State-of-the-Art Review and Future Perspectives. Front Pharmacol 12:1713

29. Gomes F et al (2021) A prospective cohort study on the safety of checkpoint inhibitors in older cancer patients - the ELDERS study. ESMO Open 6(1):100042

30. Pasello G et al (2020) Real world data in the era of Immune Checkpoint Inhibitors (ICIs): Increasing evidence and future applications in lung cancer. Cancer Treat Rev 87:102031

31. Tumeh PC et al (2017) Liver metastasis and treatment outcome with Anti-PD-1 monoclonal antibody in patients with melanoma and NSCLC. Cancer Immunol Res 5(5):417-424

32. Topalian SL et al (2019) Five-year survival and correlates among patients with advanced melanoma, renal cell carcinoma, or nonsmall cell lung cancer treated with nivolumab. JAMA Oncol 5(10): 1411-1420

33. Zhang $Y$ et al (2020) Single-cell transcriptome analysis reveals tumor immune microenvironment heterogenicity and granulocytes enrichment in colorectal cancer liver metastases. Cancer Lett 470:84-94

34. Brodt P (2016) Role of the microenvironment in liver metastasis: from pre- to prometastatic niches. Clin Cancer Res 22(24):5971-5982

35. Yu J et al (2021) Liver metastasis restrains immunotherapy efficacy via macrophage-mediated T cell elimination. Nat Med 27(1):152-164

36. Lee JC et al (2020) Regulatory T cell control of systemic immunity and immunotherapy response in liver metastasis. Sci Immunol. https://doi.org/10.1126/sciimmunol.aba0759

37. Mei Z et al (2017) Prognostic role of pretreatment blood neutrophil-to-lymphocyte ratio in advanced cancer survivors: a 
systematic review and meta-analysis of 66 cohort studies. Cancer Treat Rev 58:1-13

38. Ameratunga $\mathrm{M}$ et al (2018) Neutrophil-lymphocyte ratio kinetics in patients with advanced solid tumours on phase I trials of PD-1/ PD-L1 inhibitors. Eur J Cancer 89:56-63

39. Valero $\mathrm{C}$ et al (2021) Pretreatment neutrophil-to-lymphocyte ratio and mutational burden as biomarkers of tumor response to immune checkpoint inhibitors. Nat Commun 12(1):729

40. Templeton AJ et al (2014) Prognostic role of neutrophil-to-lymphocyte ratio in solid tumors: a systematic review and meta-analysis. J Natl Cancer Inst 106(6):dju124

41. Brandau S, Moses K, Lang S (2013) The kinship of neutrophils and granulocytic myeloid-derived suppressor cells in cancer: cousins, siblings or twins? Semin Cancer Biol 23(3):171-182

42. Sagiv JY et al (2015) Phenotypic diversity and plasticity in circulating neutrophil subpopulations in cancer. Cell Rep 10(4):562-573
43. Billan S, Kaidar-Person O, Gil Z (2020) Treatment after progression in the era of immunotherapy. Lancet Oncol 21(10):e463-e476

44. Dolladille C et al (2020) Immune Checkpoint Inhibitor Rechallenge After Immune-Related Adverse Events in Patients With Cancer. JAMA Oncol 6(6):865-871

45. Haanen J et al (2020) Rechallenge patients with immune checkpoint inhibitors following severe immune-related adverse events: review of the literature and suggested prophylactic strategy. J Immunother Cancer. https://doi.org/10.1136/jitc-2020-000604

Publisher's Note Springer Nature remains neutral with regard to jurisdictional claims in published maps and institutional affiliations.

\section{Authors and Affiliations}

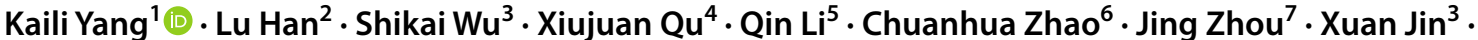 Yusheng Wang ${ }^{8}$. Dong Yan ${ }^{9} \cdot$ Zhiqiang Cheng $^{10}$. Yuwei Hua ${ }^{1}$. Yan Zhang ${ }^{11}$. Yang Ge ${ }^{12}$. Jinghua Sun ${ }^{13}$. Wei Deng ${ }^{14}$. Lin Zhao ${ }^{1}$. Yunbo Zhao ${ }^{15}$}

1 Department of Medical Oncology, Peking Union Medical College Hospital, Chinese Academy of Medical Sciences and Peking Union Medical College, Beijing 100032, China

2 Department of Medical Oncology, The General Hospital of People's Liberation Army, Beijing 100853, China

3 Department of Medical Oncology, Peking University First Hospital, Beijing 100034, China

4 Department of Medical Oncology, The First Hospital of China Medical University, Shenyang 110001, China

5 Department of Oncology, Beijing Friendship Hospital, Capital Medical University, Beijing 100050, China

6 Department of Oncology, 307 Hospital of People's Liberation Army, Beijing 100071, China

7 Department of Gastrointestinal Surgery, Peking University People's Hospital, Beijing 100044, China

8 Department of Digestive System, Shanxi Cancer Hospital, Taiyuan 030013, Shanxi, China
9 Cancer Center, Beijing Luhe Hospital, Capital Medical University, Beijing 101149, China

10 Department of Oncology of Integrative Chinese and Western Medicine, China-Japan Friendship Hospital, Beijing 100029, China

11 Department of General Surgery, Xuanwu Hospital, Capital Medical University, Beijing 100053, China

12 Department of Medical Oncology, Beijing Chaoyang Hospital, Capital Medical University, Beijing 100021, China

13 Department of Oncology, The Second Hospital of Dalian Medical University, Dalian 116044, China

14 Department of General Surgery, Beijing Friendship Hospital, Capital Medical University, Beijing 100050, China

15 Department of Oncology, Beijing Hospital, National Center of Gerontology, Beijing 100730, China 